\title{
A Terceira Missão da Universidade como Agente Tecnológico, Inovativo e Empreendedor para a Sustentabilidade: o caso da Universidade Federal do ABC
}

\author{
Carlos Antonio Medeiros Gambôa (carlos.gamboa@ufabc.edu.br) UFABC \\ Anapatrícia Morales Vilha (anapatricia.vilha@ufabc.edu.br) UFABC
}

Resumo: O desenvolvimento sustentável é uma necessidade inquestionável, reconhecida nos principais organismos internacionais e formalizada em 2015 pela Organização das Nações Unidas, na Agenda 2030. Mais do que um processo, trata-se de uma mudança cultural, uma nova forma de pensar e agir, que demandará vontade política e capacidade inovativa. A instituição melhor equipada para a inovação e a quebra de paradigmas é a universidade, em suas três missões, o Ensino, a Pesquisa e o Desenvolvimento da sociedade. O objetivo deste artigo é mostrar como as missões da universidade se inserem e atuam de modo transformador numa cultura de desenvolvimento sustentável, a partir do estudo de caso da Universidade Federal do $\mathrm{ABC}$, na região metropolitana de São Paulo.

Palavras chave: desenvolvimento sustentável, sustentabilidade, terceira missão da universidade, extensão universitária

\begin{abstract}
The sustainable development is an unquestionable requirement in nowadays, recognized in international forums and formalized in the 2030 Agenda of the United Nations Organization. Beyond a process, it is a cultural change, a new way to think and act, which will demand political will and innovative capacity. The institution best equipped to innovation and paradigm break is the University, in its three missions, Teaching, Research an Society Development. This article aims to present how the university missions insert and act in a changing manner in a sustainable development culture, using a case study of Universidade Federal do ABC, in São Paulo Metropolitan area.
\end{abstract}

Keywords: sustainable development, sustainability, third mission of university, university extension 


\section{Introdução}

O modelo de desenvolvimento percorrido no ocidente a partir da segunda metade do século XIX priorizou a expansão econômica e, com ela, a utilização intensiva de recursos naturais além da agricultura e do extrativismo, quer para geração de energia, quer como matériaprima para parques industriais, não levando em conta a degradação do ambiente, tampouco a exaustão daqueles recursos (HOBSBAWN, 1987, 1995).

A partir dos anos 1970 do século XX, o aumento da população do planeta e o fortalecimento do preceito de consumo de massa tornaram evidente que a expansão econômica não era sustentável sob o ponto de vista de reprodução do meio ambiente, o que motivou discussões em fóruns internacionais, versando sobre o efeito desse quadro sobre o gênero humano (BRUNDTLAND, 1987). Cunhou-se o conceito de desenvolvimento sustentável caracterizado pelo atendimento de necessidades e aspirações da geração atual sem comprometimento da capacidade das futuras gerações de atenderem suas correspondentes necessidades e aspirações.

Vários autores destacam o papel da inovação de produtos, processos e tecnologias, como uma das principais alavancas do desenvolvimento sustentável (ÁVILA, 2017), posicionando a mudança tecnológica e das universidades como pedras angulares para os avanços com vistas à harmonização ambiental, social, econômica, institucional e regulatória (VILHA, 2018).

Este trabalho tem o objetivo de apresentar as formas pelas quais o protagonismo da universidade se apresenta sob o contexto do desenvolvimento sustentável, destacando seu alinhamento com o que convencionamos chamar de 'Terceira Missão' da instituição, indo além das atividades de Ensino e Pesquisa. Para tanto, procedeu-se uma revisão exaustiva da literatura nos temas que concernem ao estudo, bem com a realização de um estudo de caso em profundidade da Universidade Federal do ABC (UFABC), valendo-se da análise de seus materiais institucionais, além da realização entrevistas presenciais com gestores e docentes da instituição em 2020.

\section{As Missões da Universidade}

Desde sua origem, a universidade tem atuado como centro produtor de conhecimento da sociedade humana, inicialmente concebida para a formação de quadros que amparassem intelectual e institucionalmente as estruturas de poder, como teólogos, juristas e matemáticos. Mais tarde, a instituição estendeu seu horizonte para ampliação do conhecimento sobre os 
fenômenos naturais, bem como aplicação desses conhecimentos na solução de problemas práticos (ETZKOWITZ, 2000), definidas assim a Primeira e Segunda missões da universidade, Ensino e Pesquisa.

Depois da Revolução Industrial, as universidades iniciaram atividades além de seus muros, visando a Extensão universitária de parte de seu corpo de conhecimento às populações do entorno. Nasce o eixo de Extensão (BENNEWORTH E OSBORNE, 2012), cujo objetivo primário era a transferência de conhecimento básico. Esse é o modelo embrionário do que chamaremos de Terceira Missão. FERNANDEZ-LARREA E GONZÁLEZ (2013) mostram a evolução do conceito a partir do extensionismo e sinalizam:

- Modelo tradicional, ou extensão: a instituição como fonte primária de saberes, ofertando parte destes à sociedade, de modo unidirecional e assistencialista

- Modelo economicista: a universidade como suporte tecnológico e científico do setor produtivo, transferindo conhecimento diretamente aplicável e economicamente rentável, formando e capacitando profissionais técnicos

- Modelo de desenvolvimento integral: a universidade como produtora de capital intelectual crítico, criativo e democrático, contribuindo para melhoria da qualidade de vida da sociedade.

Com suas três missões, a universidade tem suas responsabilidades não só acerca do presente em que vivemos, como na construção do futuro que nos aguarda, colaborando na definição de aspirações e possibilidades de longo prazo, transcendendo o imediato e o instrumental para se fixar no perene (RUIZ-CORBELLA, LÓPEZ-GÓMEZ, 2019).

Cumpre notar que essa mudança tem implicações culturais bastante profundas no seio do corpo docente. A instituição livre, democrática e administrada horizontalmente por pares, com foco na excelência de produção do conhecimento começa a migrar para uma propriedade de stakeholders, atendendo aos interesses econômicos e sociais de vários segmentos, como os próprios docentes, discentes, população do entorno, entidades mantenedoras e até mesmo governos (GAMBÔA 2020), ou seja, não é mais uma propriedade de uma comunidade de acadêmicos. 
Outro ponto de destaque para a Terceira Missão da universidade é a dimensão inovativa, onde a instituição tem papel relevante. Para que essa dimensão gere frutos aplicáveis e potencialmente rentáveis, é necessário que se estabeleça um processo de gestão da inovação, que envolve aspectos como direitos de propriedades intelectual, visão de empreendedorismo e até mesmo incubação de empresas. No arcabouço de conhecimento a ser desenvolvido nas universidades estão a transferência de know how, cessão de tecnologias, capacitação tecnológica de terceiros, acordos de licenciamento, pesquisa colaborativa e geração de spin offs acadêmicas (VILHA, A. et al, 2019).

Para a gestão de todas essas atividades, as universidades têm de desenvolver quadros que sejam submetidos a disciplinas específicas que os capacitem em empreendedorismo, administração econômica e mercadológica e sustentabilidade (PISANO, 2010).

Acrescente-se a isso rotinas, processos e ferramentas de gestão de tecnologia e inovação no âmbito das atividades de pesquisa e interação com atores de um sistema de inovação. As dimensões estratégicas de uma organização são i) seus processos operacionais e gerenciais - a forma como as coisas são feitas, seus padrões e rotinas, os modelos de aprendizado e execução; ii) sua posição competitiva atual - seu aporte e estoque tecnológico, seus ativos de propriedade intelectual, sua carteira de clientes e sua rede de fornecedores; iii) sua trajetória - alternativas estratégicas disponíveis, atratividade das potenciais oportunidades no horizonte (TIDD E BESSANT, 2007)

\section{O desenvolvimento sustentável e as possíveis interfaces da universidade}

A expansão da presença humana e o preenchimento de suas necessidades, especialmente econômicas, implicam num desgaste severo dos recursos naturais do planeta, bem como na degradação ambiental correspondente ao uso desses recursos e o descarte de rejeitos desse processo.

Essa perspectiva tornou-se especialmente relevante para a sociedade civil a partir dos anos 1970, especialmente pelo debate organizado pela Organização das Nações Unidas a partir de 1987, quando, após uma conferência conduzida pela primeira ministra da Noruega, Gro Harlem Brundtland, gerou-se um documento chamado Our Common Future, mais conhecido como Relatório BRUNDTLAND (1987), cujos pontos principais foram: i) estabilidade do crescimento da população; ii) garantia de segurança alimentar no longo prazo; iii) preservação da biodiversidade e dos ecossistemas, iv) diminuição do consumo de energia e desenvolvimento de 
tecnologias que privilegiem o uso de fontes renováveis; v) satisfação de todas as necessidades básicas das pessoas; vi) aumento da produção industrial nos países em desenvolvimento, baseado em tecnologias que sejam ecologicamente limpas; vii) controle da urbanização desenfreada das populações; e viii) promoção da integração entre o campo e a cidade (VILHA, 2018).

A ONU sintetizou uma agenda para desenvolvimento sustentável em 2015, os chamados Objetivos para Transformar nosso Mundo:

\section{Tabela 1: Objetivos para transformar nosso mundo}

\begin{tabular}{|c|c|c|c|}
\hline Erradicação da Pobreza & $\begin{array}{c}\text { Fome Zero e } \\
\text { Agricultura Sustentável }\end{array}$ & Saúde e Bem Estar & Educação de Qualidade \\
\hline Igualdade de Gênero & $\begin{array}{c}\text { Água Potável e } \\
\text { Saneamento }\end{array}$ & $\begin{array}{c}\text { Energia Limpa e } \\
\text { Acessível }\end{array}$ & $\begin{array}{c}\text { Trabalho Decente e } \\
\text { Crescimento Econômico }\end{array}$ \\
\hline $\begin{array}{c}\text { Indústria, Inovação e } \\
\text { Infraestrutura }\end{array}$ & $\begin{array}{c}\text { Redução de } \\
\text { Desigualdades }\end{array}$ & Cidades e Comunidades & Sustentáveis \\
\hline Ação contra Mudança \\
Global do Clima
\end{tabular}

Fonte: Horizontes 2030-A igualdade no centro do desenvolvimento sustentável (CEPAL, 2016).

A análise desses objetivos mostra que seu atingimento passa por novos modos de pensar, agir e fazer, ou seja, inovação. É visível que, nesse panorama, o papel da Universidade, especialmente em sua terceira missão, assume preponderância, por sua responsabilidade na formação e gestão de capital intelectual. Para ROJAS et al (2018) o compromisso da universidade abarca as dimensões econômica, cultural e ambiental, sem as quais não há desenvolvimento. Ela é uma instituição socialmente responsável pelos impactos causados a seu entorno, especialmente ambientais e por eles responde ante seus stakeholders (GARCIAGUTIERREZ, GAITERO, 2021)

Essa visão considera as especificidades de cada instituição em termos de perfil, local de atuação, trajetória histórica e suas interpretações acerca de sua função para a sociedade, conforme identifica a Figura 1. 


\section{Figura 1: Condicionantes para implementação da Terceira Missão nas universidades}

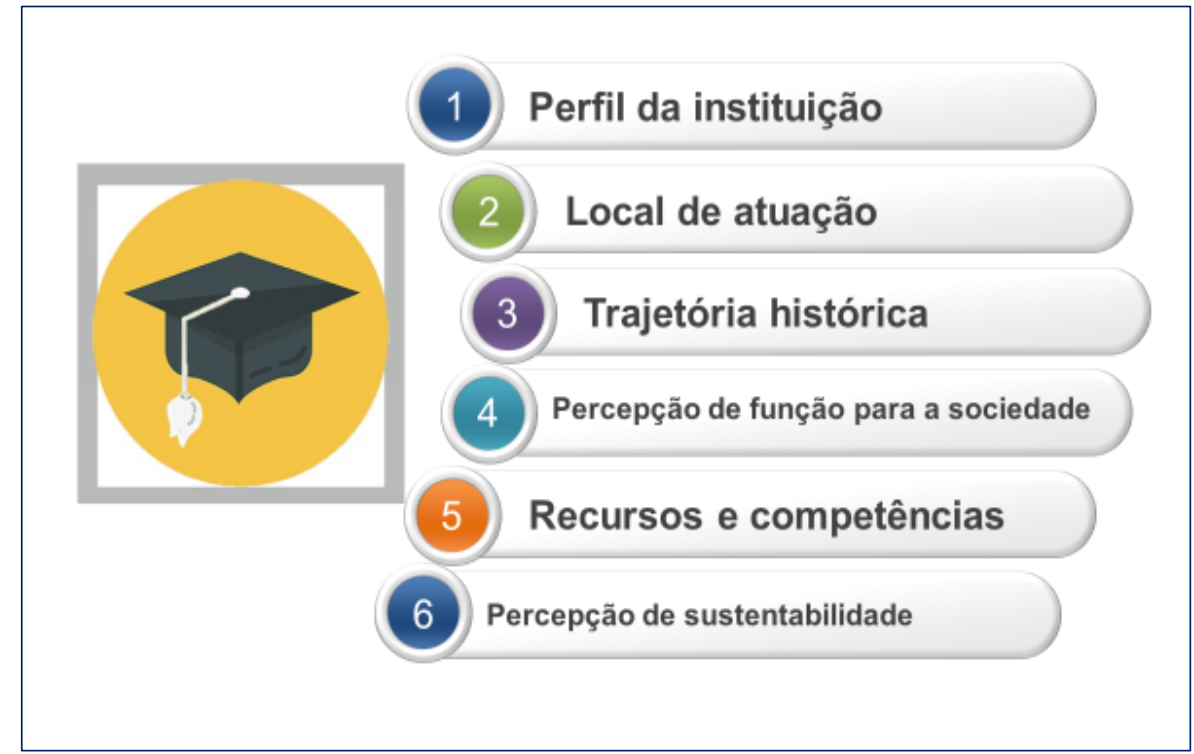

Fonte: Vilha, A.: Ciência, Tecnologia, Inovação e a Terceira Missão no Ensino Superior para o Desenvolvimento Sustentável in El Papel Estratégico de la Educación Superior em el Desarrollo Sostenible de América Latina y el Caribe, UNESCO, 2018, pp 323-344

Diversos elementos na instituição devem ser abordados e redesenhados à luz da sustentabilidade, quais sejam missão e política, curricula, pesquisa, operação dos campi, relação com a comunidade do entorno, modelo de colaboração com terceiros, prestação de contas, interdisciplinaridade, inclusão do desenvolvimento sustentável no modelo institucional e na experiência diária da população universitária, pelas vias da educação e da comunicação.

Todas as dimensões internas da universidade devem ser impressionadas: governança, comunidade acadêmica, operações, ensino e aprendizado, pesquisa e impactos extra-muros (LEAL FILHO, 2018). Dispositivos de avaliação e certificação podem ser adaptados para aplicação no ambiente universitário, pois, conforme ALSHUWAIKHAT E ABUBAKAR (2008), monitoram a redução de perdas, o desperdício de recursos e a poluição ambiental; promovem a cultura ambiental nas comunidades intra e extra acadêmicas, auxiliam gestores na busca de melhoria contínua do desempenho ambiental.

Na perspectiva do ensino (VILHA, 2018), a sustentabilidade se configura i) pela inserção ou revisão de disciplinas que enderecem o tema, transmitindo conceitos, princípios e valores, perpassando transversalmente graduação e pós graduação, ii) o envolvimento do tema pelo planejamento multidisciplinar e iii) a coleta e intercâmbio de experiências e iniciativas na 
comunidade universitária, induzindo assim a criação e desenvolvimento de competências e cultura que se traduzam em um programa de ações de sustentabilidade.

Na vertente de pesquisa (VILHA 2018), o substrato de desenvolvimento sustentável deve permear toda a estrutura física de pesquisa da universidade, bem como estar imbricado nas redes de conhecimento com as quais o capital intelectual universitário interage, nacional e internacionalmente, até o ponto em que não possa mais ser dissociado da prática científica.

A maior latitude da Terceira Missão (FERNANDEZ-LARREA, GONZALEZ, 2013) (VILHA 2018) potencializa os efeitos da implantação de uma cultura de sustentabilidade, uma vez que engloba as dimensões econômica, social e cultural, interagindo com todo um mosaico de atores. A partir do que se construiu nas dimensões Ensino e Pesquisa, a Terceira Missão responde pelo transbordo dessa cultura e corpo de conhecimento para o entorno físico, intelectual e econômico da universidade, permitindo: i) a formação de quadros disseminadores de conhecimento, ii) o desenvolvimento de aplicações práticas sustentáveis para uso e benefício da comunidade, iii) a busca e captura de recursos financeiros para implantação dessas aplicações, iv) o monitoramento dos progressos, dos resultados e a correção de rumos visando otimização dos recursos e do capital intelectual envolvidos, v) o desenvolvimento crítico da cultura de sustentabilidade, vi) um futuro sustentável.

\section{Procedimentos e técnicas de pesquisa}

Com o objetivo apresentar as formas pelas quais o protagonismo da universidade se apresenta sob o contexto do desenvolvimento sustentável, procedeu-se uma revisão exaustiva da literatura nos temas que concernem ao estudo, bem com a realização de um estudo de caso em profundidade da Universidade Federal do ABC (UFABC), valendo-se da análise de seus materiais institucionais, além da realização entrevistas presenciais com gestores e docentes da instituição em 2020.

Para analisar as práticas da terceira missão em universidades, utilizamos a tipologia sobre os Condicionantes para implementação da terceira missão nas universidades (VILHA, 2018) e que considera as especificidades de cada instituição em termos de i) perfil; ii) local de atuação; iii) trajetória histórica, e iv) suas interpretações acerca de sua função para a sociedade.

Quanto à análise das práticas com vistas ao fortalecimento do desenvolvimento sustentável nas universidades, utilizou-se como referência os Objetivos para transformar o nosso 
mundo (CEPAL, 2016). Para fins metodológicos, essas variáveis foram agrupadas em cinco grandes blocos conceituais, conforme indica a Tabela 2. O agrupamento em blocos segue o critério de associabilidade entre os objetivos.

Tabela 2: Objetivos para transformar nosso mundo

\begin{tabular}{|c|c|}
\hline Objetivo & Bloco Conceitual \\
\hline Erradicação da Pobreza & Vida \\
\hline Fome Zero e Agricultura Sustentável & Vida \\
\hline Saúde e Bem Estar & Vida \\
\hline Educação de Qualidade & Sociedade \\
\hline Igualdade de Gênero & Sociedade \\
\hline Água Potável e Saneamento & Ambiente \\
\hline Energia Limpa e Acessível & Energia \\
\hline Trabalho Decente e Crescimento Econômico & Vida \\
\hline Indústria, Inovação e Infraestrutura & Tecnologia \\
\hline Redução das Desigualdades & Sociedade \\
\hline Cidades e Comunidades Sustentáveis & Ambiente \\
\hline Consumo e Produção Responsáveis & Tecnologia \\
\hline Ação contra a Mudança Global do Clima & Ambiente \\
\hline Vida na Água & Ambiente \\
\hline Vida Terrestre & Ambiente \\
\hline Paz, Justiça e Instituições Eficazes & Sociedade \\
\hline Parcerias e Meios de Implementação & Tecnologia \\
\hline
\end{tabular}

\section{Análise e discussão dos resultados do caso da Universidade Federal do ABC (UFABC)}

\subsection{Perfil da UFABC}

"Promover o avanço do conhecimento através de ações de ensino, pesquisa e extensão, tendo como fundamentos básicos a interdisciplinaridade, a excelência e a inclusão social". Tal é a missão da Universidade Federal do ABC, fundada em 2005 para atender a uma demanda represada por educação superior pública. Apoia-se essa missão em quatro fundamentos conceituais, quais sejam: i. Ética e respeito como condições imprescindíveis para o convívio humano; ii. Excelência acadêmica, abrangendo excelência em pesquisa, ensino, extensão e gestão; iii. Interdisciplinaridade, considerada como efetiva interação entre as áreas do 
conhecimento; iv. Inclusão social, praticada tanto como ato de responsabilidade e solidariedade como um processo ativo de identificação e desenvolvimento de talentos (PDI UFABC 20132022).

Adicionalmente, entre seus quatro fundamentos operacionais, observa-se a busca por "Inovação acadêmica como forma de atender às mudanças da sociedade e da tecnologia" e "Responsabilidade ambiental".

\subsection{Local de atuação e linha do tempo de criação das principais áreas e iniciativa ligadas à} terceira missão para a sustentabilidade na UFABC

Produzimos uma análise de documentos e iniciativas realizadas pela instituição, tendo em vista que a terceira missão enfeixa atividades de inovação e extensão, e que estão, organizacionalmente, distribuídas na UFABC em duas áreas distintas, quais sejam, a Agência de Inovação e a Pró-reitoria de Extensão (PROEC). A linha do tempo, a seguir (Figura 2) mostra os marcos principais:

20062010

Fonte: Elaboração própria

\subsection{Trajetória histórica das ações ligadas à terceira missão para a sustentabilidade na} UFABC

Criada em 2010, a Agência de Inovação (InovaUFABC) tem como missão a busca de desenvolvimento econômico sustentado através da evolução tecnológica e estímulo à postura empreendedora, contribuindo com as atividades de ensino, pesquisa e extensão. Ela está dividida em seis divisões técnicas: empreendedorismo tecnológico, inteligência estratégica, propriedade intelectual, transferência de tecnologia, incubadoras e parques tecnológicos e extensão tecnológica/formação continuada (InovaUFABC, 2021).

Verifica-se que, além da gestão da propriedade intelectual, a agência desenvolve atividades de capacitação da população universitária, especialmente nos quesitos empreendedorismo e gestão organizacional, bem como promove a interação entre a universidade 
e o meio empresarial, não só na disseminação de conhecimento e tecnologia, como na capacitação de interlocutores.

Neste trabalho, foram elencadas as ações da Inova ABC em termos de patentes aprovadas no INPI, projetos conduzidos junto a CNPq e FAPESP, acordos de incubação de empresas e projetos conjuntos com o meio empresarial. Essas ações foram agrupadas nos Blocos Conceituais supra-mencionados, em função de seu alinhamento com a perspectiva de Desenvolvimento Sustentável, conforme resultados sintetizados na Tabela 3 a seguir.

Tabela 3: Distribuição das ações da InovaUFABC por blocos conceituais dos “Objetivos para transformar nosso mundo" de CEPAL (2016)

\begin{tabular}{|c|c|c|c|c|c|}
\hline Bloco Conceitual & $\begin{array}{c}\text { Patentes } \\
\text { INPI }\end{array}$ & $\begin{array}{c}\text { Projetos } \\
\text { CNPq }\end{array}$ & $\begin{array}{l}\text { Projetos } \\
\text { FAPESP }\end{array}$ & $\begin{array}{c}\text { Bolsas } \\
\text { FAPESP }\end{array}$ & $\begin{array}{l}\text { Total } \\
\text { Bloco }\end{array}$ \\
\hline Ambiente & 6 & 15 & 9 & 21 & 51 \\
\hline Energia & 9 & 14 & 10 & 9 & 42 \\
\hline Sociedade & 2 & 8 & 6 & 3 & 19 \\
\hline Tecnologia & 9 & 8 & 5 & 9 & 31 \\
\hline Vida & 18 & 15 & 18 & 37 & 88 \\
\hline Total Sustentabilidade & 44 & 60 & 48 & 79 & 231 \\
\hline Total Projetos & 49 & 76 & 98 & 132 & 355 \\
\hline
\end{tabular}

Fonte: Autoria Própria (2019).

A InovaUFABC tem incubadas treze empresas, das quais doze embutem desenvolvimento sustentável. Firmou também 74 acordos com o setor empresarial e entidades governamentais, compreendendo consultoria, transferência tecnológica e capacitação, onde 48 destes acordos envolvem sustentabilidade, conforme metodologia de análise desenvolvida neste trabalho e estão dispostas em:

- Ambiente: 10 .

- Energia: 6.

- Tecnologia: 14 
- Vida: 18/

No que tange ao processo de desenvolvimento de projetos de inovação sob a perspectiva da sustentabilidade, a literatura da área tem mostrado a influência dos stakeholders (fornecedores primários e intermediários, governo, clientes, sociedade) nas fases de desenvolvimento e avaliação dos projetos de P\&D (MILLER, 1995; TIPPING et al, 1995; COOMBS et al, 1998), oferecendo uma forma de analisar a interferência dos princípios do desenvolvimento sustentável sobre a gestão de projetos de inovação. Como preconizado por CLARK e WHEELWRIGHT (1993) sobre o sucesso de projetos formados estar condicionado à presença de times multidisciplinares, a UFABC tem em sua política institucional iniciativas com o intuito de reunir diferentes competências para viabilizar o desenvolvimento de projetos de inovação (incluindo os relacionados à sustentabilidade mencionados acima) à luz dessa percepção.

Verificamos que o trabalho da InovaUFABC endereça de modo consistente a Terceira Missão da universidade, tendo o desenvolvimento sustentável como pano de fundo constante. Serão avaliadas a seguir as atividades de Extensão, que completam o envolvimento da instituição com a sociedade.

Em via paralela, as atividades de extensão da UFABC são executadas por sua Pró-reitoria de Extensão (PROEC) (PROEC, 2021). Para esta análise, foram selecionadas as atividades vinculadas a essa área da UFABC, realizadas em 2019, compreendendo 120 cursos e 146 projetos, conforme indica Tabela 4 a seguir.

Tabela 4: Distribuição das ações da PROEC por blocos conceituais dos “Objetivos para transformar nosso mundo" CEPAL (2016)

\begin{tabular}{|c|c|c|c|}
\hline Bloco Conceitual & Cursos & Projetos & Total Bloco \\
\hline Ambiente & 10 & 30 & 40 \\
\hline Energia & 1 & & 1 \\
\hline Sociedade & 48 & 43 & 91 \\
\hline Tecnologia & 14 & 22 & 36 \\
\hline Vida & 7 & 15 & 22 \\
\hline
\end{tabular}




\begin{tabular}{|c|c|c|c|}
\hline Total Sustentabilidade & 80 & 110 & 190 \\
\hline Total Ações & 120 & 146 & 266 \\
\hline
\end{tabular}

Fonte: Autoria Própria (2019).

A soma das ações realizadas pela InovaUFABC e pela PROEC mostram o equilíbrio da participação com foco direto no Ser Humano (Sociedade e Vida), sem desdenhar os aspectos do entorno (Ambiente, Energia e Tecnologia), como mostra a Tabela 5 a seguir.

Tabela 5: Síntese das ações InovaUFABC e da PROEC por blocos conceituais dos “Objetivos para transformar nosso mundo" de CEPAL (2016)

\begin{tabular}{|c|c|c|c|c|}
\hline Bloco Conceitual & Eixo & Inova & Extensão & $\begin{array}{c}\text { Total } \\
\text { Bloco }\end{array}$ \\
\hline Ambiente & Entorno & 51 & 40 & 91 \\
\hline Energia & Entorno & 42 & 1 & 43 \\
\hline Sociedade & Humano & 19 & 91 & 110 \\
\hline Tecnologia & Entorno & 31 & 36 & 67 \\
\hline Vida & Humano & 88 & 22 & 110 \\
\hline Fonte: Autoria Própria (2019). & & 231 & 190 & 421 \\
\hline
\end{tabular}

\subsection{Interpretações acerca da função da UFABC para a sociedade}

Desenvolvimento Sustentável, mais que um conjunto de ações, é uma mudança cultural, e como tal, deve ser construída a cada dia, até que se transforme em parte indivisível do indivíduo e da sociedade. Com essa visão, a UFABC, a partir de 2015, desenvolveu e implantou um Plano de Logística Sustentável. A Tabela 6 a seguir apresenta uma visão global do projeto, dividido em sete frentes de atuação:

\section{Tabela 6: Síntese do Projeto de Logística Sustentável}




\begin{tabular}{|c|c|}
\hline Grupo de Trabalho & Objetivos \\
\hline - Água e esgoto & $\begin{array}{l}\text { - } \text { Redução do consumo de água potável } \\
\text { - } \text { Tratamento e reuso de águas pluviais e esgotos } \\
\text { - } \quad \text { Manutenção preventiva de infra-estrutura } \\
\text { - }\end{array}$ \\
\hline - Energia & $\begin{array}{l}\text { - Racionalizar o uso e fontes de energia } \\
\text { - Supervisionar continuamente equipamentos visando potencial } \\
\text { modernização tecnológica e ganho de eficiência }\end{array}$ \\
\hline $\begin{array}{l}\text { - Material de consumo e } \\
\text { licitações sustentáveis }\end{array}$ & $\begin{array}{l}\text { - Otimizar o consumo de materiais } \\
\text { - Definir modelo analítico para avaliação de licitações sob a ótica da } \\
\text { sustentabilidade }\end{array}$ \\
\hline - Gerenciamento de resíduos & - Gerir coleta, separação e gestão de resíduos \\
\hline - Espaços & - Melhoria de qualidade de vida nos espaços da universidade \\
\hline $\begin{array}{l}\text { - Deslocamento e mobilidade } \\
\text { urbana }\end{array}$ & $\begin{array}{l}\text { - Aprimoramento dos sistemas de deslocamento, especialmente intra e } \\
\text { intercampi } \\
\text { - Viabilização de sistemas de deslocamento sustentáveis }\end{array}$ \\
\hline - Implementação e divulgação & $\begin{array}{l}\text { - Atuar como agente facilitador da implementação do programa, } \\
\text { através de comunicação e conscientização da população universitária, } \\
\text { e fornecedores } \\
\text { - Auxiliar na construção e permanência da cultura de sustentabilidade }\end{array}$ \\
\hline
\end{tabular}

Em complemento, a análise dos curricula de graduação da instituição mostrou 188 disciplinas diretamente relacionadas ao Desenvolvimento Sustentável, abertas a todos os alunos da universidade.

\section{Considerações finais}

O Desenvolvimento Sustentável é uma necessidade inquestionável. Entretanto, como mudança de paradigma, exige esforços no sentido de sua inserção na sociedade que hoje parecem insuficientes.

A Agenda 2030, subscrita por 193 países, bem como os diversos fóruns internacionais de discussão sobre o assunto, tem imposto pressão institucional e senso de urgência ao assunto, bem como estabelecido eixos conceituais claros, representados pelos Dezessete Objetivos para Transformar Nosso Mundo, estabelecidos como princípios daquela agenda. Uma mudança cultural implica em novas formas de abordar, de agir e de pensar a realidade, que poderíamos sintetizar no conceito Inovação. 
Doutra parte, em se tratando de inovar e promover mudanças culturais, nenhuma instituição é mais eficaz que uma Universidade, por sua capacidade de desenvolver e disseminar novos saberes, evidente em suas três missões, quais sejam, o Ensino, a Pesquisa e o Desenvolvimento da sociedade, nas dimensões cultural, social e econômica.

Neste trabalho, buscamos mostrar o quanto as práticas para o Desenvolvimento Sustentável estão alinhadas e imbricadas nas missões da Universidade Federal do ABC, na região metropolitana de São Paulo.

Verificamos que o Desenvolvimento Sustentável é objeto de parcela substancial dos esforços da instituição em suas três missões. A terceira missão, que implica no desenvolvimento do entorno, é exercida, na UFABC, por dois órgãos diretamente subordinados à reitoria, quais sejam, a Pró-Reitoria de Extensão (PROEC), cujas atividades abarcam as dimensões social e cultural, e a Agência de Inovação (Inova $\mathrm{ABC}$ ), que cuida da inovação tecnológica produzida pela universidade e seu transbordo para o meio empresarial. Ambas seguem o Plano de Desenvolvimento Institucional (PDI) da universidade, que tem como um de seus pilares a sustentabilidade. Adicionalmente, todos os cursos de graduação ministrados têm disciplinas relacionadas ao Desenvolvimento Sustentável em seus curricula, implicando numa formação cultural sólida que percorre transversalmente a graduação e a pós-graduação.

Este trabalho abre espaço para o aprofundamento do estudo da UFABC, considerando a evolução dos processos ao longo do tempo e o desenvolvimento e aplicação de métricas para avaliação dessa evolução.

\section{Referências}

ALSHUWAIKHAT, H., ABUBAKAR, I.: An integrated approach to achieving campus sustainability: assessment of the current campus environmental management practices in Journal of Cleaner Production 16 (2008).

ÁVILA, L. ET AL: Barriers to innovation and sustainability at universities around the world, in Journal of Cleaner Production 164 (2017).

BENNEWORTH, P.,OSBORNE, M.: Knowledge, Engagement and Higher Education in Europe in Global University Network (2014). 
BESSANT, J. TIDD, J. Innovation and entrepreneurship. John Wiley \& Sons, 2007, capítulo 2, pp. 39-73.

BRUNDTLAND, G.: Our Common Future - Call for Action in Environmental Conservation, Vol. 14, No. 4, Winter 1987-Journal of the Foundation for Environmental Conservation.

CEPAL, HORIZONTES 2030-A igualdade no centro do desenvolvimento sustentável, 2016, $36^{\circ}$ Período de seções da Cepal.

CLARK, K.B.; WHEELWRIGHT, S.C. Managing new product and process development: text and cases. New York: The Free Press, 1993.

COOMBS, R., MCMEEKIN, A. PYBUS, R. Toward the development of benchmarking tools for R\&D project management. R\&D Management, 1998, 28, 3, 175-186.

ETZKOVITZ,H. ET AL: The future of university and the university of the future: evolution of ivory tower to entrepreneurial paradigm, in Research Policy 29, 2000. Pp 313-330.

FERNANDEZ-LARREA, M.; GONZÁLEZ, C. Extensión universitaria, proyección social o tercera misión? una reflexión necesaria, in Revista Congreso Universidad. Vol. II, No. 2, 2013, ISSN: 2306-918X.

GAMBÔA, C. A Terceira Missão nas universidades federais brasileiras: Conceitos e modelo de implementação, Dissertação de Mestrado, UFABC, 2020 
GARCIA-GUTIERREZ, J.;GAITERO, C.: Las políticas supranacionales de educación superior ante la «tercera misión» de la Universidad: el caso del aprendizaje-servicio in Revista Española de Educación Comparada | núm. 37 (enero-junio 2021), pp. 256-280

HOBSBAWM, E, The Age of Empire, Weindenfeld \& Nicolson, 1987,São Paulo, Brasil: Companhia das Letras.

InovaUFABC - Agência de Inovação da UFABC. Apresentação institucional. Disponível em http://inova.ufabc.edu.brconsultadoem 30.05.2021.

LEAL FILHO, W. ET AL, Sustainable development policies as indicators and preconditions for sustainability efforts at universities: Fact or fiction? In International Journal of Sustainability in Higher Education Vol. 19 No. 1, 2018 pp. 85-113.

MILLER, W. L. A Broader mission of R\&D. Research Technology Management, 1995, 38, $6,24-36$.

PISANO, G.: The Evolution of Science-Based Business: Innovating How We Innovate, in Harvard Business School, Working Paper 10-062, 2010

PROEC - Apresentação da Pró-Reitoria de Extensão, Disponível em https://proec.ufabc.edu.br/?source=Portal, consultado em 30.05.2012.

ROJAS,M. et al: La tercera misión en la universidad: evolución y diversas actividades XXIII Congreso Anual de Contaduria, Administración y Informática, Ciudad de México, 2018

RUIZ-CORBELLA, M, LÓPEZ-GÓMEZ E.: La misión de la universidad en el siglo xxi: comprender su origen para proyectar su futuro in Revista de la Educación Superior 48 (189) (2019) 1-19 13 
TIPPING, J. W., ZEFFREN, E. FUSFELD, A. R. Assessing the value of technology

UNIVERSIDADE FEDERAL DO ABC, Plano de Desenvolvimento Institucional 2013-2022.

VILHA, A.: Ciência, Tecnologia, Inovação e a Terceira Missão no Ensino Superior para o Desenvolvimento Sustentável in El Papel Estratégico de la Educación Superior en el Desarrollo Sostenible de América Latina y el Caribe, UNESCO, 2018, pp 323-344.

VILHA, A. et al: Interação Universidade-Empresa e trilha metodológica para gestão de parcerias in Boas Práticas de Gestão em Núcleos de inovação Tecnológica, Experiências Inovadoras, vol 2, FORTEC, Edições Brasil 2019 